\title{
Efficiency of High-Order Accurate Difference Schemes for the Korteweg-de Vries Equation
}

\author{
Kanyuta Poochinapan, ${ }^{1}$ Ben Wongsaijai, ${ }^{1}$ and Thongchai Disyadej ${ }^{2}$ \\ ${ }^{1}$ Department of Mathematics, Faculty of Science, Chiang Mai University, Chiang Mai 50200, Thailand \\ ${ }^{2}$ Electricity Generating Authority of Thailand, Phitsanulok 65000, Thailand \\ Correspondence should be addressed to Kanyuta Poochinapan; kanyuta@hotmail.com
}

Received 5 August 2014; Accepted 2 November 2014; Published 8 December 2014

Academic Editor: Igor Andrianov

Copyright (C) 2014 Kanyuta Poochinapan et al. This is an open access article distributed under the Creative Commons Attribution License, which permits unrestricted use, distribution, and reproduction in any medium, provided the original work is properly cited.

Two numerical models to obtain the solution of the KdV equation are proposed. Numerical tools, compact fourth-order and standard fourth-order finite difference techniques, are applied to the KdV equation. The fundamental conservative properties of the equation are preserved by the finite difference methods. Linear stability analysis of two methods is presented by the Von Neumann analysis. The new methods give second- and fourth-order accuracy in time and space, respectively. The numerical experiments show that the proposed methods improve the accuracy of the solution significantly.

\section{Introduction}

Researchers in the past have worked on mathematical models explaining the behavior of a nonlinear wave phenomenon which is one of the significant areas of applied research. Derived by Korteweg and de Vries [1], the Korteweg-de Vries eqaution ( $\mathrm{KdV}$ equation) is one of the mathematical models which are used to study a nonlinear wave phenomenon. The $\mathrm{KdV}$ equation has been used in very wide applications, such as magnetic fluid waves, ion sound waves, and longitudinal astigmatic waves.

The KdV equation has been solved numerically by various methods, such as the collocation method [2-4], the finite element method $[5,6]$, the Galerkin method [7-10], the spectral method $[11,12]$, and the finite difference method [1318]. To create a numerical tool, the finite difference method for the KdV equation is developed until now. Zhu [13] solved the KdV equation using the implicit difference method. The scheme is unconditionally linearly stable and has a truncation error of order $O\left(\tau+h^{2}\right)$. Qu and Wang [14] developed the alternating segment explicit-implicit (ASE-I) difference scheme consisting of four asymmetric difference schemes, a classical explicit scheme, and an implicit scheme, which is unconditionally linearly stable by the analysis of linearization procedure. Wang et al. [15] have proposed an explicit finite difference scheme for the KdV equation. The scheme is more stable than the Zabusky-Kruskal (Z-K) scheme [16] when it is used to simulate the collisions of multisoliton. The stability of the method in [15] was also discussed by using the frozen coefficient Von Neumann analysis method. The time step limitation of the method in [15] is twice looser than that of the Z-K method. Moreover, Kolebaje and Oyewande [17] investigated the behavior of solitons generated from the KdV equation that depends on the nature of the initial condition, by using the Goda method [18], the Z-K method, and the Adomian decomposition method.

The stability, accuracy, and efficiency, which are in conflict with each other, are the desired properties of the finite difference scheme. Implicit approximation is requested in order to reach the stability of the finite difference scheme. A high-order accuracy in the spatial discretization is desired in various problems. The stencil becomes wider with increasing order of accuracy for a high-order method of a conventional scheme. Furthermore, using an implicit method results in the solution of an algebraic system for equations with extensive bandwidth. It is required to improve schemes that have a broad range of stability and high order of accuracy. Additionally, this leads to the solution of the system for linear 
equations with a pentadiagonal matrix, that is, the system of linear equations arising from a standard second-order discretization of a boundary value problem. A method to conquer the conflict between stability, accuracy, and computational cost is the development of a high-order compact scheme.

In recent decades, many scientists concentrated upon the difference method that makes a discrete analogue effective in the fundamental conservation properties. This causes us to create finite difference schemes which preserve the mass and energy of solutions for the $\mathrm{KdV}$ equation. In this paper, two fourth-order difference schemes are constructed for the one dimensional KdV equation:

$$
u_{t}+\alpha u_{x x x}+\gamma\left(u^{2}\right)_{x}=0, \quad x_{L}<x<x_{R}, 0 \leq t \leq T,
$$

with an initial condition

$$
u(x, 0)=u_{0}(x), \quad x_{L} \leq x \leq x_{R},
$$

and boundary conditions

$$
\begin{aligned}
& u\left(x_{L}, t\right)=u\left(x_{R}, t\right)=0, \\
& u_{x}\left(x_{L}, t\right)=u_{x}\left(x_{R}, t\right)=0, \\
& u_{x x}\left(x_{L}, t\right)=u_{x x}\left(x_{R}, t\right)=0, \\
& 0 \leq t \leq T,
\end{aligned}
$$

where $\alpha$ and $\gamma$ are any real number. When $-x_{L} \gg 0$ and $x_{R} \gg$ 0 , the initial-boundary value problem (1)-(3) is consistent, so the boundary condition (3) is reasonable. By assumptions, the solitary wave solution and its derivatives have the following asymptotic values, $u \rightarrow 0$ as $x \rightarrow \pm \infty$, and for $n \geq 1$, $\partial^{n} u / \partial x^{n} \rightarrow 0$ as $x \rightarrow \pm \infty$. Moreover, we obtain the solution properties as follows [19]:

$$
\begin{aligned}
& I_{1}=\int_{x_{L}}^{x_{R}} u(x, t) d x, \\
& I_{2}=\int_{x_{L}}^{x_{R}} u(x, t)^{2} d x, \\
& I_{3}=\int_{x_{L}}^{x_{R}}\left[2 \gamma u(x, t)^{3}-3 \alpha\left[u(x, t)_{x}\right]^{2}\right] .
\end{aligned}
$$

The content of this paper is organized as follows. In the next section, we create fourth-order finite difference schemes for the KdV equation with the initial and boundary conditions. The stability of finite difference schemes is discussed and the conservative approximations are also given. The results on validation of finite difference schemes are presented in Section 3, where we make a detailed comparison with available data, to confirm and illustrate our theoretical analysis. Finally, we finish our paper by conclusions in the last section.

\section{Difference Schemes}

We start the discussion of finite difference schemes by defining a grid of points in the $(x, t)$ plane. For simplicity, we use a uniform grid for a discrete process with states identified by $x_{j}=x_{L}+j h$ which the grid size is $h=\left(x_{R}-x_{L}\right) / M$, where $M$ is the number of grid points. Therefore, the grid will be the points $\left(x_{j}, t_{n}\right)=\left(x_{L}+j h, n \tau\right)$ for arbitrary integers $j$ and $n$. Here $\tau$ is a time increment (time step length). We write the notation $u_{j}^{n}$ for a value of a function $u$ at the grid point $\left(x_{L}+j h, n \tau\right)$.

In this paper, we give a complete description of our finite difference schemes and an algorithm for the formulation of the problem (1)-(3). We use the following notations for simplicity:

$$
\begin{gathered}
\bar{u}_{j}^{n}=\frac{u_{j}^{n+1}+u_{j}^{n-1}}{2}, \quad\left(u_{j}^{n}\right)_{\hat{t}}=\frac{u_{j}^{n+1}-u_{j}^{n-1}}{2 \tau}, \\
\left(u_{j}^{n}\right)_{\bar{x}}=\frac{u_{j}^{n}-u_{j-1}^{n}}{h}, \quad\left(u_{j}^{n}\right)_{x}=\frac{u_{j+1}^{n}-u_{j}^{n}}{h}, \\
\left(u_{j}^{n}\right)_{\widehat{x}}=\frac{u_{j+1}^{n}-u_{j-1}^{n}}{2 h}, \quad\left(u_{j}^{n}\right)_{\ddot{x}}=\frac{u_{j+2}^{n}-u_{j-2}^{n}}{4 h}, \\
\left(u^{n}, v^{n}\right)=h \sum_{j=1}^{M-1} u_{j}^{n} v_{j}^{n}, \quad\left\|u^{n}\right\|^{2}=\left(u^{n}, u^{n}\right), \\
\left\|u^{n}\right\|_{\infty}=\max _{1 \leq j \leq M-1}\left|u_{j}^{n}\right| .
\end{gathered}
$$

As introduced in the following subsections, the techniques for determining the value of numerical solution to (1) are used.

2.1. Compact Fourth-Order Finite Difference Scheme. By setting $w=-\alpha u_{x x x}-\gamma\left(u^{2}\right)_{x}$, (1) can be written as $w=u_{t}$. By the Taylor expansion, we obtain

$$
\begin{aligned}
w_{j}^{n}= & \left(\partial_{t} u\right)_{j}^{n}=\left(u_{j}^{n}\right)_{\widehat{t}}+O\left(\tau^{2}\right), \\
w_{j}^{n}= & -\alpha\left[\left(u_{j}^{n}\right)_{x \bar{x} \hat{x}}-\frac{h^{2}}{4}\left(\partial_{x}^{5} u\right)_{j}^{n}\right] \\
& -\gamma\left[\left[\left(u_{j}^{n}\right)^{2}\right]_{\widehat{x}}-\frac{h^{2}}{6}\left(\partial_{x}^{3} u^{2}\right)_{j}^{n}\right]+O\left(h^{4}\right) .
\end{aligned}
$$

From (6), we have

$$
\alpha\left(\partial_{x}^{5} u\right)_{j}^{n}=-\gamma\left(\partial_{x}^{3} u^{2}\right)_{j}^{n}-\left(\partial_{x}^{2} w\right)_{j}^{n} .
$$

Substituting (8) into (7), we get

$$
\begin{aligned}
w_{j}^{n}= & -\alpha\left(u_{j}^{n}\right)_{x \bar{x} \hat{x}}-\frac{h^{2}}{4}\left(\partial_{x}^{2} w\right)_{j}^{n}-\gamma\left[\left(u_{j}^{n}\right)^{2}\right]_{\hat{x}} \\
& -\frac{h^{2}}{12} \gamma\left(\partial_{x}^{3} u^{2}\right)_{j}^{n}+O\left(h^{4}\right) .
\end{aligned}
$$

Using second-order accuracy for approximation, we obtain

$$
\begin{aligned}
& \left(\partial_{x}^{3} u^{2}\right)_{j}^{n}=\left[\left(u_{j}^{n}\right)^{2}\right]_{x \bar{x} \bar{x}}+O\left(h^{2}\right), \\
& \left(\partial_{x}^{2} w\right)_{j}^{n}=\left(w_{j}^{n}\right)_{x \bar{x}}+O\left(h^{2}\right) .
\end{aligned}
$$


The following method is the proposed compact finite difference scheme to solve the problem (1)-(3):

$$
\begin{aligned}
\left(u_{j}^{n}\right)_{\widehat{t}} & +\frac{h^{2}}{4}\left(u_{j}^{n}\right)_{x \bar{x} \hat{t}}+\alpha\left(\bar{u}_{j}^{n}\right)_{x \bar{x} \hat{x}}+\gamma\left[\left(u_{j}^{n}\right)\left(\bar{u}_{j}^{n}\right)\right]_{\widehat{x}} \\
& +\frac{\gamma h^{2}}{12}\left[\left(u_{j}^{n}\right)\left(\bar{u}_{j}^{n}\right)\right]_{x \bar{x} \hat{x}}=0,
\end{aligned}
$$

where

$$
u_{j}^{0}=u_{0}\left(x_{j}\right), \quad 0 \leq j \leq M .
$$

Since the boundary conditions are homogeneous, they give

$$
u_{0}^{n}=u_{M}^{n}=0, \quad\left(u_{0}^{n}\right)_{\widehat{x}}=\left(u_{M}^{n}\right)_{\widehat{x}}=0, \quad 1 \leq n \leq N .
$$

At this time, let $e_{j}^{n}=v_{j}^{n}-u_{j}^{n}$ where $v_{j}^{n}$ and $u_{j}^{n}$ are the solution of (1)-(3) and (11)-(13), respectively. Then, we obtain the following error equation:

$$
\begin{aligned}
r_{j}^{n}= & \left(e_{j}^{n}\right)_{\hat{t}}+\frac{h^{2}}{4}\left(e_{j}^{n}\right)_{x \bar{x} \hat{t}}+\alpha\left(\bar{e}_{j}^{n}\right)_{x \bar{x} \hat{x}}+\gamma\left[\left(v_{j}^{n}\right)\left(\bar{v}_{j}^{n}\right)\right]_{\widehat{x}} \\
& -\gamma\left[\left(u_{j}^{n}\right)\left(\bar{u}_{j}^{n}\right)\right]_{\widehat{x}}+\frac{\gamma h^{2}}{12}\left[\left(v_{j}^{n}\right)\left(\bar{v}_{j}^{n}\right)\right]_{x \bar{x} \hat{x}} \\
& -\frac{\gamma h^{2}}{12}\left[\left(u_{j}^{n}\right)\left(\bar{u}_{j}^{n}\right)\right]_{x \bar{x} \widehat{x}}
\end{aligned}
$$

where $r_{j}^{n}$ denotes the truncation error. By using the Taylor expansion, it is easy to see that $r_{j}^{n}=O\left(\tau^{2}+h^{4}\right)$ holds as $\tau, h \rightarrow$ 0 .

The Von Neumann stability analysis of (11) with $u_{j}^{n}=$ $\xi^{n} e^{i k j h}$, where $i^{2}=-1$ and $k$ is a wave number, gives the following the amplification factor:

$$
\xi^{2}=\frac{A-i \tau B}{A+i \tau B}
$$

where

$$
\begin{aligned}
A= & 6 h^{3}(\cos (k h)+1), \\
B= & 12 \alpha(\sin (2 k h)-2 \sin (k h)) \\
& +\gamma h^{2}\left(u_{j}^{n}\right)(\sin (4 k h)+10 \sin (2 k h)) .
\end{aligned}
$$

The amplification factor which is a complex number has its modulus equal to one; therefore the compact finite difference scheme is unconditionally stable.

Theorem 1. Suppose $u(x, t)$ is smooth enough, then the scheme (11)-(13) is conservative in a sense:

$$
I_{1}^{n}=\frac{h^{M}}{2} \sum_{j=1}^{M-1}\left(u_{j}^{n+1}+u_{j}^{n}\right)=I_{1}^{n-1}=\cdots=I_{1}^{0},
$$

under assumptions $u_{1}=u_{M-1}=0$.
Proof. By multiplying (11) by $h$, summing up for $j$ from 1 to $M-1$, and considering the boundary condition and assuming $u_{1}=u_{M-1}=0$, we get

$$
\frac{h}{2 \tau} \sum_{j=1}^{M-1}\left(u_{j}^{n+1}-u_{j}^{n-1}\right)=0 .
$$

Then, this gives (17).

2.2. Standard Fourth-Order Finite Difference Scheme. By the fact $\left(u^{2}\right)_{x}=(2 / 3)\left[u u_{x}+\left(u^{2}\right)_{x}\right]$ and using an implicit finite difference method, we propose a standard seven-point implicit difference scheme for the problem (1)-(3):

$$
\begin{aligned}
& \left(u_{j}^{n}\right)_{\widehat{t}}+\alpha\left(\frac{3}{2}\left(\bar{u}_{j}^{n}\right)_{x \bar{x} \hat{x}}-\frac{1}{2}\left(\bar{u}_{j}^{n}\right)_{x \bar{x} \ddot{x}}\right) \\
& \quad+2 \gamma\left[\frac{4}{9}\left(\left(u_{j}^{n} \bar{u}_{j}^{n}\right)_{\widehat{x}}+u_{j}^{n}\left(\bar{u}_{j}^{n}\right)_{\widehat{x}}\right)-\frac{1}{9}\left(\left(u_{j}^{n} \bar{u}_{j}^{n}\right)_{\ddot{x}}+u_{j}^{n}\left(\bar{u}_{j}^{n}\right)_{\ddot{x}}\right)\right] \\
& =0
\end{aligned}
$$

where

$$
u_{j}^{0}=u_{0}\left(x_{j}\right), \quad 0 \leq j \leq M
$$

Since the boundary conditions are homogeneous, we obtain

$$
\begin{gathered}
u_{0}^{n}=u_{M}^{n}=0, \\
4\left(u_{0}^{n}\right)_{\bar{x}}-\left(u_{0}^{n}\right)_{\ddot{x}}=4\left(u_{M}^{n}\right)_{\bar{x}}-\left(u_{M}^{n}\right)_{\ddot{x}}=0, \\
-\left(u_{-1}^{n}\right)_{x \bar{x}}+14\left(u_{0}^{n}\right)_{x \bar{x}}-\left(u_{1}^{n}\right)_{x \bar{x}} \\
=-\left(u_{M-1}^{n}\right)_{x \bar{x}} 14\left(u_{M}^{n}\right)_{x \bar{x}}-\left(u_{M+1}^{n}\right)_{x \bar{x}} \\
=0, \quad 1 \leq n \leq N .
\end{gathered}
$$

$u, u_{x}$, and $u_{x x}$ are required by the standard fourth-order technique to be zero at the upstream and downstream boundaries because the method utilizes a seven-point finite difference scheme for the approximation of solution $u$. Through the analytical technique of contrasting, (11) requires two homogeneous boundary conditions only.

Now, let $e_{j}^{n}=v_{j}^{n}-u_{j}^{n}$ where $v_{j}^{n}$ and $u_{j}^{n}$ are the solution of (1)-(3) and (19)-(22), respectively. Then, we obtain the following error equation:

$$
\begin{aligned}
\left(e_{j}^{n}\right)_{\hat{t}} & +\alpha \frac{3}{2}\left(\bar{e}_{j}^{n}\right)_{x \bar{x} \hat{x}}-\alpha \frac{1}{2}\left(\bar{e}_{j}^{n}\right)_{x \bar{x} \ddot{x}} \\
& +\frac{8 \gamma}{9}\left[\left(\left(v_{j}^{n} \bar{v}_{j}^{n}\right)_{\widehat{x}}+v_{j}^{n}\left(\bar{v}_{j}^{n}\right)_{\widehat{x}}\right)-\left(\left(u_{j}^{n} \bar{u}_{j}^{n}\right)_{\widehat{x}}+u_{j}^{n}\left(\bar{u}_{j}^{n}\right)_{\widehat{x}}\right)\right] \\
& -\frac{2 \gamma}{9}\left[\left(\left(v_{j}^{n} \bar{v}_{j}^{n}\right)_{\ddot{x}}+v_{j}^{n}\left(\bar{v}_{j}^{n}\right)_{\ddot{x}}\right)-\left(\left(u_{j}^{n} \bar{u}_{j}^{n}\right)_{\ddot{x}}+u_{j}^{n}\left(\bar{u}_{j}^{n}\right)_{\ddot{x}}\right)\right]=0,
\end{aligned}
$$

where $r_{j}^{n}$ denotes the truncation error. By using the Taylor expansion, it is easy to see that $r_{j}^{n}=O\left(\tau^{2}+h^{4}\right)$ holds as $\tau, h \rightarrow$ 0 . 
The Von Neumann stability analysis of (19) with $u_{j}^{n}=$ $\xi^{n} e^{i k j h}$ gives the following amplification factor:

$$
\xi^{2}=\frac{36 h^{3}-i \tau A}{36 h^{3}+i \tau A},
$$

where

$$
\begin{aligned}
A= & 4 \gamma h^{2}\left(u_{j}^{n}\right)(-\sin (4 k h)+7 \sin (2 k h)+8 \sin (k h)) \\
& +9 \alpha(-\sin (3 k h)+8 \sin (2 k h)-13 \sin (k h)) .
\end{aligned}
$$

The amplification factor which is a complex number has its modulus equal to one; therefore the finite difference scheme is unconditionally stable.

Theorem 2. Suppose $u(x, t)$ is smooth enough, then the scheme (11)-(13) is conservative in a sense:

$$
\begin{aligned}
I_{1}^{n}= & \frac{h^{M}}{2-1} \sum_{j=1}^{n+1}\left(u_{j}^{n+1}+u_{j}^{n}\right) \\
& +\tau h \gamma \sum_{j=1}^{M-1}\left[\frac{4}{9} u_{j}^{n}\left(u_{j}^{n+1}\right)_{\widehat{x}}-\frac{1}{9} u_{j}^{n}\left(u_{j}^{n+1}\right)_{\ddot{x}}\right] \\
= & I_{1}^{n-1}=\cdots=I_{1}^{0},
\end{aligned}
$$

under assumptions $u_{1}=u_{2}=u_{M-2}=u_{M-1}=0$. Moreover, the scheme (19)-(22) is conservative in a sense:

$$
I_{2}^{n}=\frac{1}{2}\left\|u^{n}\right\|^{2}+\frac{1}{2}\left\|u^{n+1}\right\|^{2}=I_{2}^{n-1}=\cdots=I_{2}^{0} .
$$

Proof. By multiplying (11) by $h$, summing up for $j$ from 1 to $M-1$, and considering the boundary condition and assuming $u_{1}=u_{2}=u_{M-2}=u_{M-1}=0$, we have

$$
\begin{aligned}
\tau h \sum_{j=1}^{M-1}\left[\frac{8}{9}\left(u_{j}^{n}\left(\bar{u}_{j}^{n}\right)_{\widehat{x}}\right)-\frac{2}{9}\left(u_{j}^{n}\left(\bar{u}_{j}^{n+1}\right)_{\ddot{x}}\right)\right] \\
=\tau h \sum_{j=1}^{M-1}\left[\frac{4}{9}\left(u_{j}^{n}\left(u_{j}^{n+1}\right)_{\widehat{x}}-u_{j}^{n-1}\left(u_{j}^{n}\right)_{\widehat{x}}\right)\right. \\
\left.-\frac{1}{9}\left(u_{j}^{n}\left(u_{j}^{n+1}\right)_{\ddot{x}}-u_{j}^{n-1}\left(u_{j}^{n}\right)_{\ddot{x}}\right)\right] .
\end{aligned}
$$

As a result, we have

$$
\begin{aligned}
& \frac{h}{2} \sum_{j=1}^{M-1}\left(u_{j}^{n+1}-u_{j}^{n-1}\right) \\
&+\gamma \tau h \sum_{j=1}^{M-1}\left[\frac{4}{9}\left(u_{j}^{n}\left(u_{j}^{n+1}\right)_{\widehat{x}}-u_{j}^{n-1}\left(u_{j}^{n}\right)_{\widehat{x}}\right)\right. \\
&\left.\quad-\frac{1}{9}\left(u_{j}^{n}\left(u_{j}^{n+1}\right)_{\ddot{x}}-u_{j}^{n-1}\left(u_{j}^{n}\right)_{\ddot{x}}\right)\right]=0 .
\end{aligned}
$$

Then, this gives (27). We then take an inner product between (19) and $2 \bar{u}^{n}$. We obtain

$$
\begin{aligned}
\frac{1}{2 \tau}\left(\left\|u^{n+1}\right\|^{2}-\left\|u^{n-1}\right\|^{2}\right)+\frac{3 \alpha}{2}\left(\left(\bar{u}^{n}\right)_{x \bar{x} \bar{x}},\left(\bar{u}^{n}\right)\right) \\
-\frac{\alpha}{2}\left(\left(\bar{u}^{n}\right)_{x \bar{x} \ddot{x}},\left(\bar{u}^{n}\right)\right)+2 \gamma\left(\varphi^{n}\left(u^{n}, \bar{u}^{n}\right), \bar{u}^{n}\right)=0,
\end{aligned}
$$

where

$$
\begin{aligned}
\varphi^{n}\left(u_{j}^{n}, \bar{u}_{j}^{n}\right)= & \frac{4}{9}\left(\left(u_{j}^{n} \bar{u}_{j}^{n}\right)_{\widehat{x}}+u_{j}^{n}\left(\bar{u}_{j}^{n}\right)_{\widehat{x}}\right) \\
& -\frac{1}{9}\left(\left(u_{j}^{n} \bar{u}_{i}^{n}\right)_{\ddot{x}}+u_{j}^{n}\left(\bar{u}_{j}^{n}\right)_{\ddot{x}}\right),
\end{aligned}
$$

by considering the boundary condition (13). According to

$$
\begin{aligned}
& \left(\bar{u}_{x \bar{x} \bar{x}}^{n}, \bar{u}^{n}\right)=0, \\
& \left(\bar{u}_{x \bar{x} \ddot{x}}^{n}, \bar{u}^{n}\right)=0,
\end{aligned}
$$

indeed,

$$
\begin{aligned}
\left(\varphi^{n}\left(u^{n}, \bar{u}^{n}\right), \bar{u}^{n}\right) & \\
= & \frac{4 h}{9} \sum_{j=1}^{M-1}\left[u_{j}^{n}\left(u_{j}^{n+1}\right)_{\widehat{x}}+\left(u_{j}^{n} u_{j}^{n+1}\right)_{\widehat{x}}\right] u_{j}^{n+1} \\
& -\frac{h}{9} \sum_{j=1}^{M-1}\left[u_{j}^{n}\left(u_{j}^{n+1}\right)_{\ddot{x}}+\left(u_{j}^{n} u_{j}^{n+1}\right)_{\ddot{x}}\right] u_{j}^{n+1} \\
= & \frac{2}{9} \sum_{j=1}^{M-1}\left[\left(u_{j}^{n} u_{j}^{n+1} u_{j+1}^{n+1}-u_{j-1}^{n} u_{j-1}^{n+1} u_{j}^{n+1}\right)\right. \\
& \left.+\left(u_{j+1}^{n} u_{j}^{n+1} u_{j+1}^{n+1}-u_{j}^{n} u_{j-1}^{n+1} u_{j}^{n+1}\right)\right] \\
& -\frac{1}{36} \sum_{j=1}^{M-1}\left[\left(u_{j}^{n} u_{j}^{n+1} u_{j+2}^{n+1}-u_{j-2}^{n} u_{j-2}^{n+1} u_{j}^{n+1}\right)\right. \\
& \left.+\left(u_{j+2}^{n} u_{j}^{n+1} u_{j+2}^{n+1}-u_{j}^{n} u_{j-2}^{n+1} u_{j}^{n+1}\right)\right] \\
= & 0 .
\end{aligned}
$$

Therefore,

$$
\frac{1}{2 \tau}\left(\left\|u^{n+1}\right\|^{2}-\left\|u^{n-1}\right\|^{2}\right)=0
$$

Then, this gives (28).

A conservative approximation confirms that the energy would not increase in time, which allows making the scheme stable.

\section{Numerical Experiments}

In this section, we present numerical experiments on the classical KdV equation when $\alpha=1$ and $\gamma=3$ with both difference schemes. The accuracy of the methods is measured 
TABLE 1: Error and convergence rate of the compact finite difference scheme (11) at $t=60, h=0.5$, and $\tau=0.25$.

\begin{tabular}{lccc}
\hline & $\tau, h$ & $\tau / 4, h / 2$ & $\tau / 16, h / 4$ \\
\hline$\|e\|$ & $1.39538 \times 10^{-2}$ & $7.15872 \times 10^{-4}$ & $4.49013 \times 10^{-5}$ \\
Rate & - & 4.28481 & 3.99487 \\
$\|e\|_{\infty}$ & $7.64991 \times 10^{-3}$ & $3.32024 \times 10^{-4}$ & $2.08869 \times 10^{-5}$ \\
Rate & - & 4.52608 & 3.99062 \\
\hline
\end{tabular}

TABLE 2: Error and convergence rate of the standard fourth-order finite difference scheme (19) at $t=60, h=0.5$, and $\tau=0.25$.

\begin{tabular}{lccc}
\hline & $\tau, h$ & $\tau / 4, h / 2$ & $\tau / 16, h / 4$ \\
\hline$\|e\|$ & $1.59924 \times 10^{-1}$ & $9.79739 \times 10^{-3}$ & $6.09352 \times 10^{-4}$ \\
Rate & - & 4.02885 & 4.00705 \\
$\|e\|_{\infty}$ & $8.63999 \times 10^{-2}$ & $5.33149 \times 10^{-3}$ & $3.33067 \times 10^{-4}$ \\
Rate & - & 4.01842 & 4.00066 \\
\hline
\end{tabular}

TABLE 3: Invariants of $I_{1}, I_{2}$, and $I_{3}$ of the compact fourth-order finite difference scheme (11).

\begin{tabular}{lccc}
\hline$t$ & $I_{1}$ & $I_{2}$ & $I_{3}$ \\
\hline 0 & 2.0000000000 & 0.6666666667 & 1.2058836346 \\
10 & 1.9999449243 & 0.6666680888 & 1.2059201473 \\
20 & 2.0001106778 & 0.6666680896 & 1.2059186978 \\
30 & 1.9999055324 & 0.6666679386 & 1.2059155167 \\
40 & 2.0001880153 & 0.6666680804 & 1.2059193791 \\
50 & 1.9999670401 & 0.6666680255 & 1.2059262538 \\
60 & 1.9998768932 & 0.6666679688 & 1.2059162036 \\
\hline
\end{tabular}

by the comparison of numerical solutions with the exact solutions as well as other numerical solutions from methods in the literatures, by using $\|\cdot\|$ and $\|\cdot\|_{\infty}$ norm. The initial conditions for each problem are chosen in such a way that the exact solutions can be explicitly computed. In case $\alpha=1$ and $\gamma=3$, the $\mathrm{KdV}$ equation has the analytical solution as

$$
u(x, t)=0.5 \operatorname{sech}^{2}(0.5(x-t)) .
$$

Therefore, the initial condition of (1) takes the form

$$
u_{0}(x)=0.5 \operatorname{sech}^{2}(0.5(x))
$$

For these particular experiments, we set $x_{L}=-40$, $x_{R}=100$, and $T=60$. We make a comparison between the compact fourth-order finite difference scheme (11) and the standard fourth-order finite difference scheme (19). So, the results on this experiment in terms of errors at the time $t=60$ is reported in Tables 1 and 2, respectively. It is clear that the results obtained by the compact fourth-order difference scheme (11) are more accurate than the ones obtained by the standard fourth-order difference scheme but the estimation of the rate of convergence for both schemes is close to the theoretically predicted fourth-order rate of convergence. It can be seen that the computational efficiency of the scheme (11) is better than that of the scheme (19), in terms of error.

Conservative approximation, that is a supplementary constraint, is essential for a suitable difference equation to make a discrete analogue effective to the fundamental conservation properties of the governing equation. Then, we can calculate three conservative approximations by using discrete forms as follows:

$$
\begin{aligned}
& I_{1} \approx \frac{h}{2} \sum_{j=1}^{M}\left(u_{j}^{n+1}+u_{j}^{n}\right), \\
& I_{2} \approx \frac{h}{2} \sum_{j=1}^{M}\left[\left(u_{j}^{n+1}\right)^{2}+\left(u_{j}^{n}\right)^{2}\right], \\
& I_{3} \approx h \sum_{j=1}^{M}\left[2 \gamma\left(\frac{\left(u_{j}^{n+1}\right)^{3}+\left(u_{j}^{n}\right)^{3}}{2}\right)\right. \\
& \left.-3 \alpha\left(\frac{\left(u_{j}^{n+1}\right)_{\widehat{x}}^{2}+\left(u_{j}^{n}\right)_{\widehat{x}}^{2}}{2}\right)\right] .
\end{aligned}
$$

Here, we take $h=0.25$ and $\tau=h^{2}$ at $t \in[0,60]$ for the compact fourth-order finite difference scheme (11) and the standard fourth-order finite difference scheme (19) and results are presented in Tables 3 and 4, respectively. The numerical results show that both two schemes can preserve the discrete conservation properties.

The second-order explicit scheme (Z-K scheme) and the second-order implicit scheme (Goda scheme) are used for testing the numerical performance of the new schemes. In Figure 1, we see that the $\mathrm{Z}-\mathrm{K}$ scheme computes reasonable solutions using $h=0.1$ and $\tau=0.01$, except that the approximate solution at $t=0.1$ does not maintain the shape of the exact solution. Similar calculations at $t=0.1$ and $t=0.11$ 
TABLE 4: Invariants of $I_{1}, I_{2}$, and $I_{3}$ of the standard fourth-order finite difference scheme (19).

\begin{tabular}{lccc}
\hline$t$ & $I_{1}$ & $I_{2}$ & $I_{3}$ \\
\hline 0 & 2.0000000000 & 0.6666666667 & 1.2058836346 \\
10 & 2.0000527573 & 0.6666666667 & 1.2059115241 \\
20 & 2.0000219448 & 0.6666666667 & 1.2059125783 \\
30 & 1.9999931738 & 0.6666666667 & 1.2059105915 \\
40 & 2.0001264687 & 0.6666666667 & 1.2059099477 \\
50 & 1.9999456225 & 0.6666666667 & 1.2059116281 \\
60 & 1.9998875333 & 0.6666666667 & 1.2059106816 \\
\hline
\end{tabular}

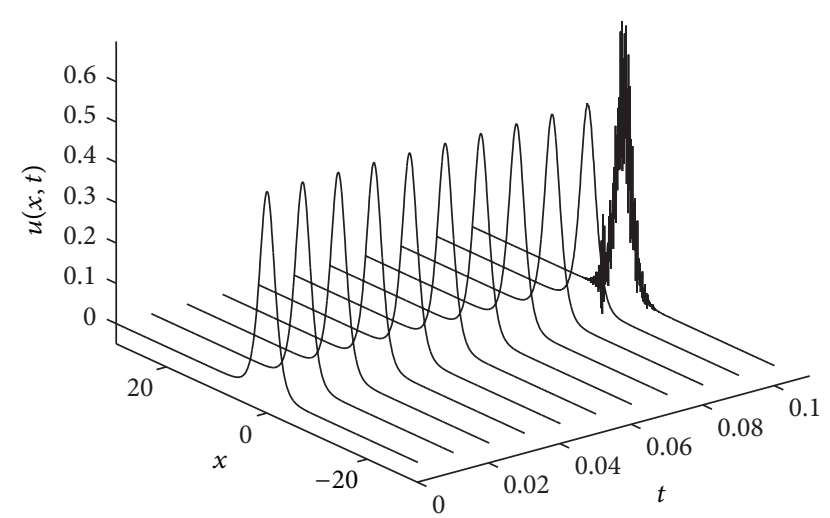

FIgURE 1: Explicit solutions using the Z-K scheme at $t \in[0,0.1]$, $x_{L}=-40, x_{R}=100, h=0.1$, and $\tau=0.01$.

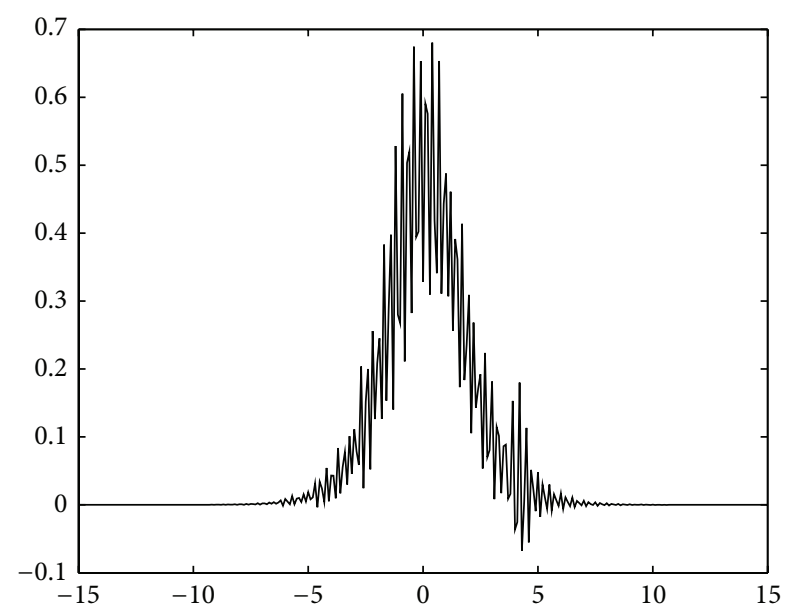

Figure 2: Explicit solution using the Z-K scheme at 10 time steps, $x_{L}=-40, x_{R}=100, h=0.1$, and $\tau=0.01$.

are demonstrated in Figures 2 and 3, respectively. The figures show that numerical waveforms begin to oscillate at $t=0.1$ and show a blowup when $t=0.11$. According to the results, the $\mathrm{Z}-\mathrm{K}$ scheme is numerically unstable, regardless of how small time increment is.

As shown in Figure 2, the results of the Z-K scheme are greatly fluctuating at 10 time steps. Therefore, It can not be used to predict the behavior of the solution at long time. Figures 4 and 5 present the numerical solutions by using the

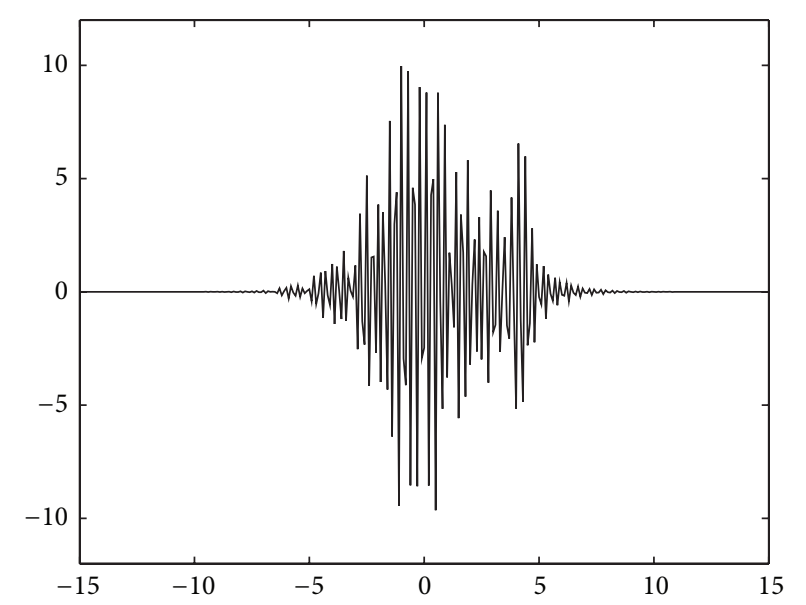

Figure 3: Explicit solution using the Z-K scheme at 11 time steps, $x_{L}=-40, x_{R}=100, h=0.1$, and $\tau=0.01$.

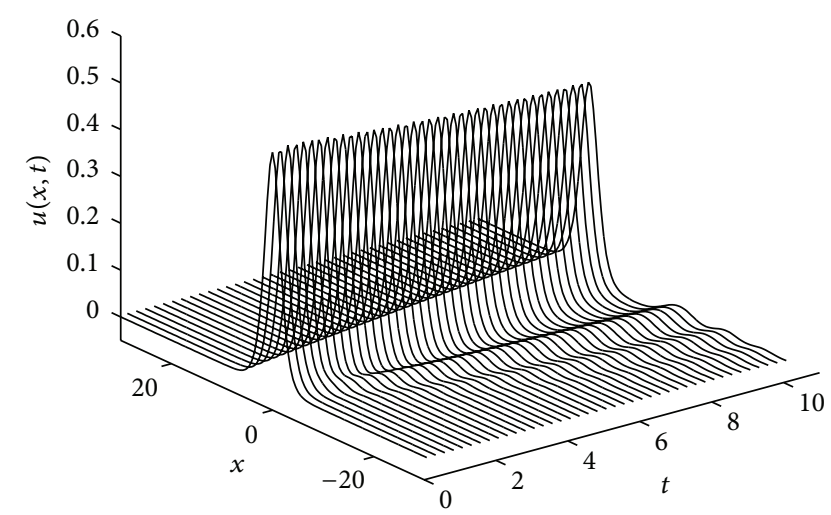

FIgURE 4: Implicit solutions using the Goda scheme at $t \in[0,10]$, $x_{L}=-40, x_{R}=100, h=0.5$, and $\tau=0.25$.

Goda scheme. We see that the Goda scheme can run very well at $h=0.5$ and $\tau=0.25$. However, the result is still slightly oscillate at the left side of the solution.

Using the same parameters as the Goda scheme, Figures 6 and 7 present waveforms with $t \in[0,10]$. The result obtained by the fourth-order difference schemes is greatly improved, compared to that obtained by the second-order schemes.

Figure 8 shows the numerical solution at $t=200$. The result from the compact fourth-order difference scheme (11) is almost perfectly sharp. From the point of view for the long 


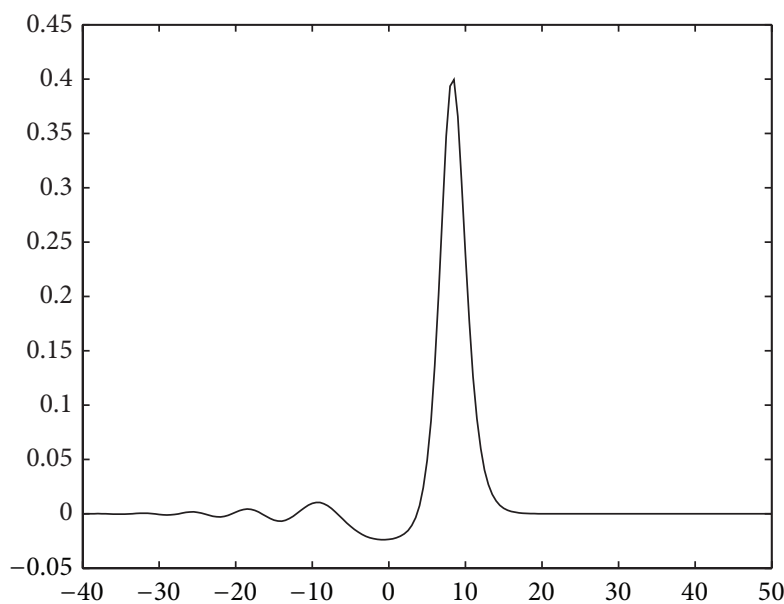

Figure 5: Implicit solution using the Goda scheme at $t=10, x_{L}=$ $-40, x_{R}=100, h=0.5$, and $\tau=0.25$.

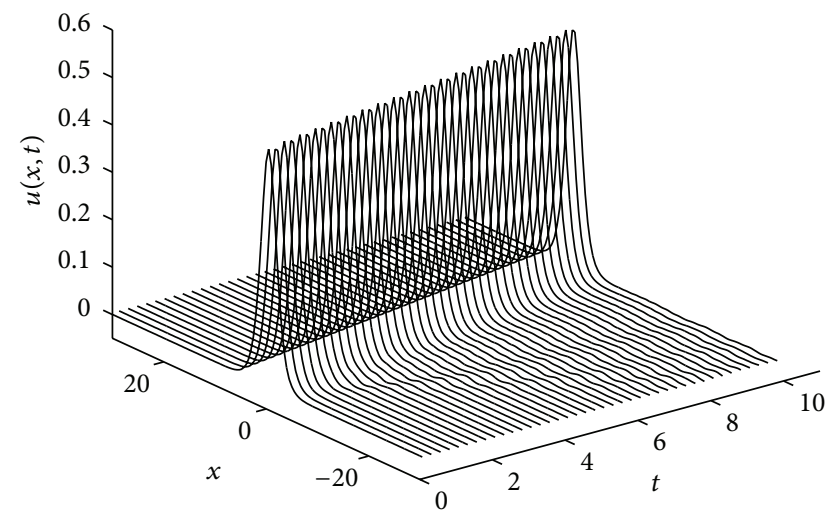

Figure 6: Numerical solutions using the scheme (11) at $t \in[0,10]$, $x_{L}=-40, x_{R}=100, h=0.5$, and $\tau=0.25$.

time behavior of the resolution, the compact fourth-order difference scheme (11) can be seen to be much better than the standard implicit fourth-order scheme (19).

The results of this section suffice to claim that both numerical implementations offer a valid approach toward the numerical investigation of a solution of the KdV equation, especially for the compact finite difference method.

\section{Conclusion}

Two conservative finite difference schemes for the KdV equation are introduced and analyzed. The construction of the compact finite difference scheme (11) requires only a regular five-point stencil at higher time level, which is similar to the standard second-order Crank-Nicolson scheme, the explicit scheme [16], and the implicit scheme [18]. However, the construction of the standard fourth-order scheme (19) requires a seven-point stencil at higher time level. The accuracy and stability of the numerical schemes for the solutions of the KdV

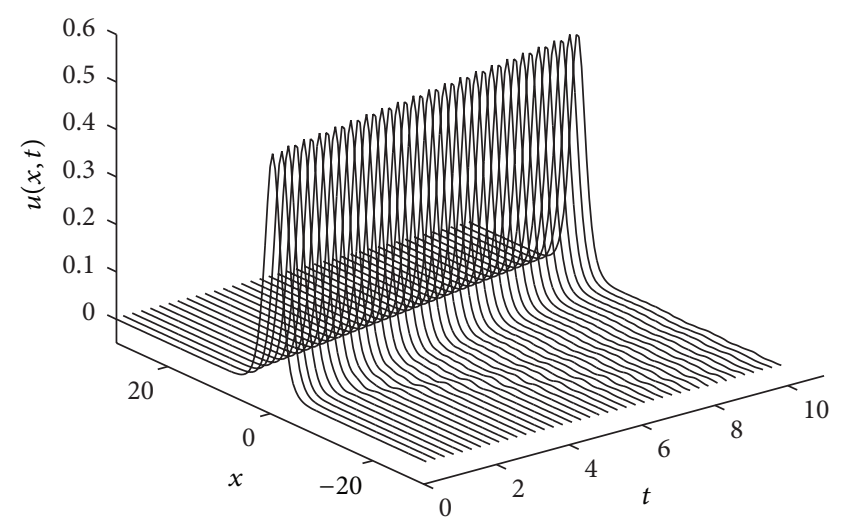

FIgURE 7: Numerical solutions using the scheme (19) at $t \in[0,10]$, $x_{L}=-40, x_{R}=100, h=0.5$, and $\tau=0.25$.

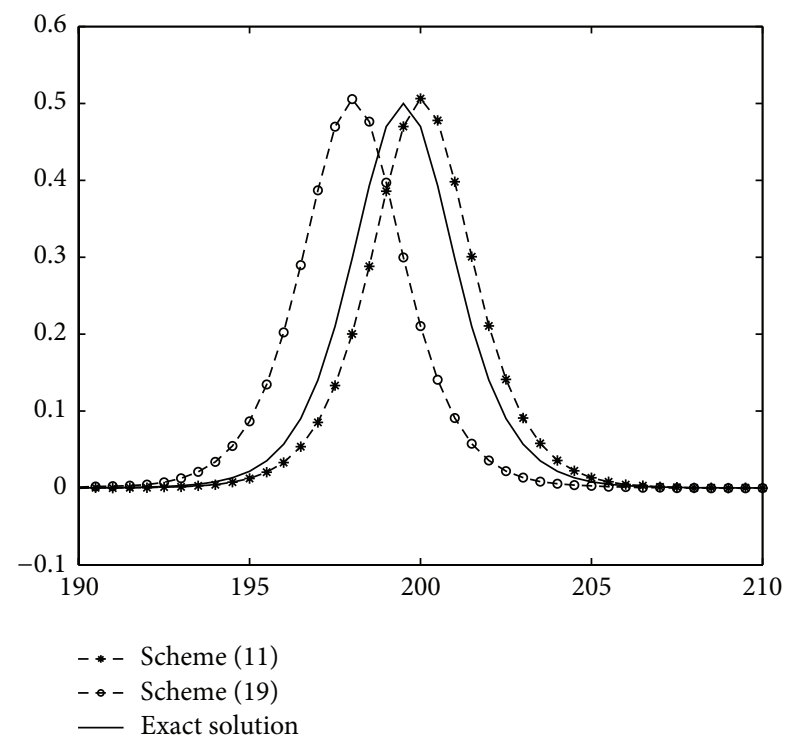

FIGURE 8: Numerical solutions at $t=200, x_{L}=-40, x_{R}=300$, $h=0.5$, and $\tau=0.25$.

equation can be tested by using the exact solution. In the paper, the numerical experiments show that the present methods support the analysis of convergence rate. The performance of the fourth-order schemes is well efficient at long time by comparing with the second-order schemes $[16,18]$.

\section{Conflict of Interests}

The authors declare that there is no conflict of interests regarding the publication of this paper.

\section{Acknowledgment}

This research was supported by Chiang Mai University.

\section{References}

[1] D. J. Korteweg and G. de Vries, "On the change of form of long waves advancing in a rectangular canal and on a new type of 
long stationary wave," Philosophical Magazine, vol. 39, pp. 422449, 1895.

[2] D. Pavoni, "Single and multidomain Chebyshev collocation methods for the Korteweg-de Vries equation," Calcolo, vol. 25, no. 4, pp. 311-346, 1988.

[3] A. A. Soliman, "Collocation solution of the Korteweg-de Vries equation using septic splines," International Journal of Computer Mathematics, vol. 81, no. 3, pp. 325-331, 2004.

[4] H. Kalisch and X. Raynaud, "On the rate of convergence of a collocation projection of the KdV equation," Mathematical Modelling and Numerical Analysis, vol. 41, no. 1, pp. 95-110, 2007.

[5] G. F. Carey and Y. Shen, "Approximations of the KdV equation by least squares finite elements," Computer Methods in Applied Mechanics and Engineering, vol. 93, no. 1, pp. 1-11, 1991.

[6] L. R. T. Gardner, G. A. Gardner, and A. H. A. Ali, "Simulations of solitons using quadratic spline finite elements," Computer Methods in Applied Mechanics and Engineering, vol. 92, no. 2, pp. 231-243, 1991.

[7] M. E. Alexander and J. L. Morris, "Galerkin methods applied to some model equations for non-linear dispersive waves," Journal of Computational Physics, vol. 30, no. 3, pp. 428-451, 1979.

[8] S. R. Barros and J. W. Cárdenas, "A nonlinear Galerkin method for the shallow-water equations on periodic domains," Journal of Computational Physics, vol. 172, no. 2, pp. 592-608, 2001.

[9] H. Ma and W. Sun, "A Legendre-Petrov-Galerkin and Chebyshev collocation method for third-order differential equations," SIAM Journal on Numerical Analysis, vol. 38, no. 5, pp. 14251438, 2000.

[10] J. Shen, "A new dual-Petrov-Galerkin method for third and higher odd-order differential equations: application to the KDV equation," SIAM Journal on Numerical Analysis, vol. 41, no. 5, pp. 1595-1619, 2003.

[11] W. Heinrichs, "Spectral approximation of third-order problems," Journal of Scientific Computing, vol. 14, no. 3, pp. 275-289, 1999.

[12] Y. Maday and A. Quarteroni, "Error analysis for spectral approximation of the Korteweg-de Vries equation," Modélisation Mathématique et Analyse numérique, vol. 22, no. 3, pp. 499-529, 1988.

[13] S. Zhu, "A scheme with a higher-order discrete invariant for the KdV equation," Applied Mathematics Letters, vol. 14, no. 1, pp. 17-20, 2001.

[14] F.-l. Qu and W.-q. Wang, "Alternating segment explicit-implicit scheme for nonlinear third-order KdV equation," Applied Mathematics and Mechanics. English Edition, vol. 28, no. 7, pp. 973980, 2007.

[15] H.-P. Wang, Y.-S. Wang, and Y.-Y. Hu, "An explicit scheme for the KdV equation," Chinese Physics Letters, vol. 25, no. 7, pp. 2335-2338, 2008.

[16] N. J. Zabusky and M. D. Kruskal, "Interaction of "solitons" in a collisionless plasma and the recurrence of initial states," Physical Review Letters, vol. 15, no. 6, pp. 240-243, 1965.

[17] O. Kolebaje and O. Oyewande, "Numerical solution of the Korteweg-de Vries equation by finite differenece an d adomain decomposition method," International Journal of Basic and Applied Sciences, vol. 1, no. 3, pp. 321-335, 2012.

[18] K. Goda, "On stability of some finite difference schemes for the Korteweg-de Vries equation," Journal of the Physical Society of Japan, vol. 39, no. 1, pp. 229-236, 1975.

[19] S. Hamdi, W. H. Enright, W. E Schiesser, and J. J. Gottlieb, "Exact solutions and conservation laws for coupled generalized
Korteweg-de Vries and quintic regularized long wave equations," Nonlinear Analysis, Theory, Methods and Applications, vol. 63, no. 5-7, pp. e1425-e1434, 2005. 


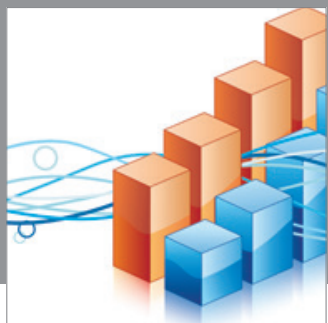

Advances in

Operations Research

mansans

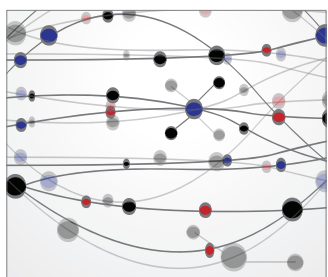

The Scientific World Journal
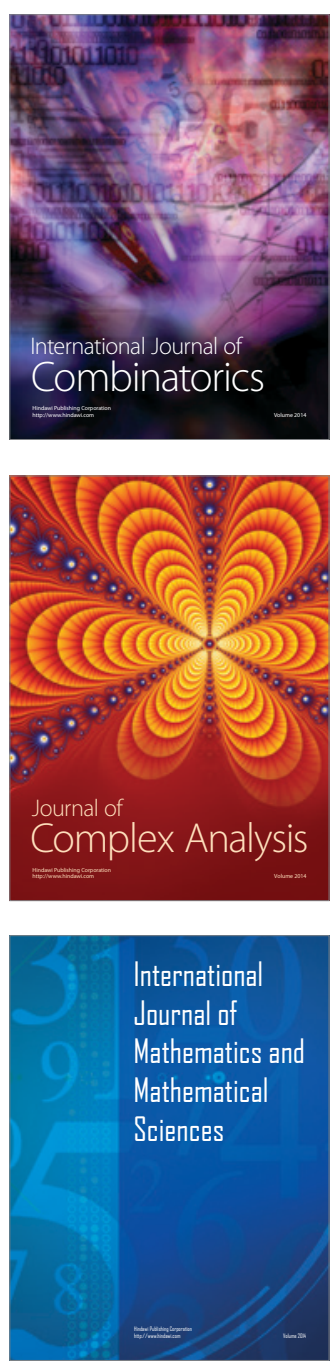
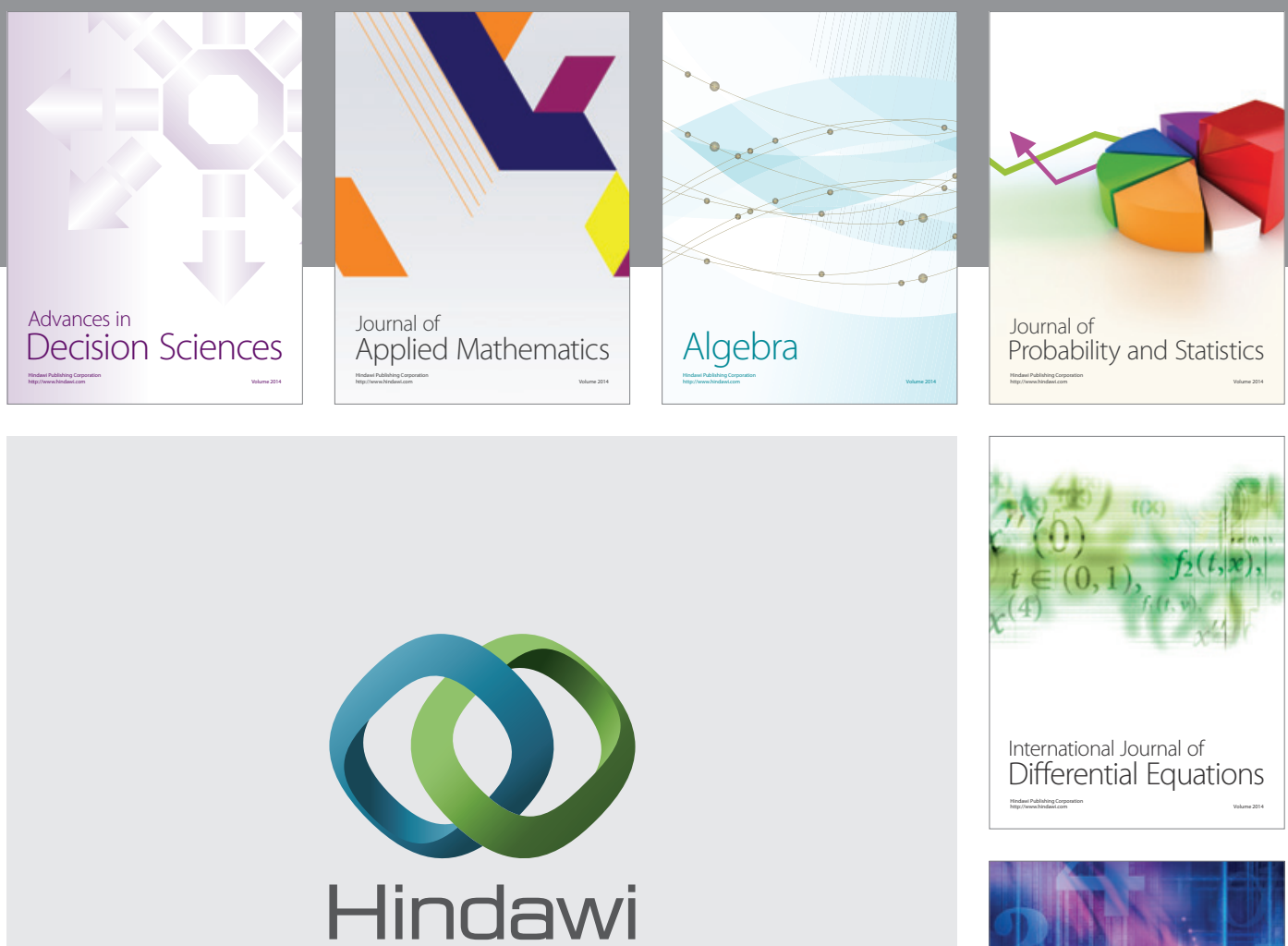

Submit your manuscripts at http://www.hindawi.com
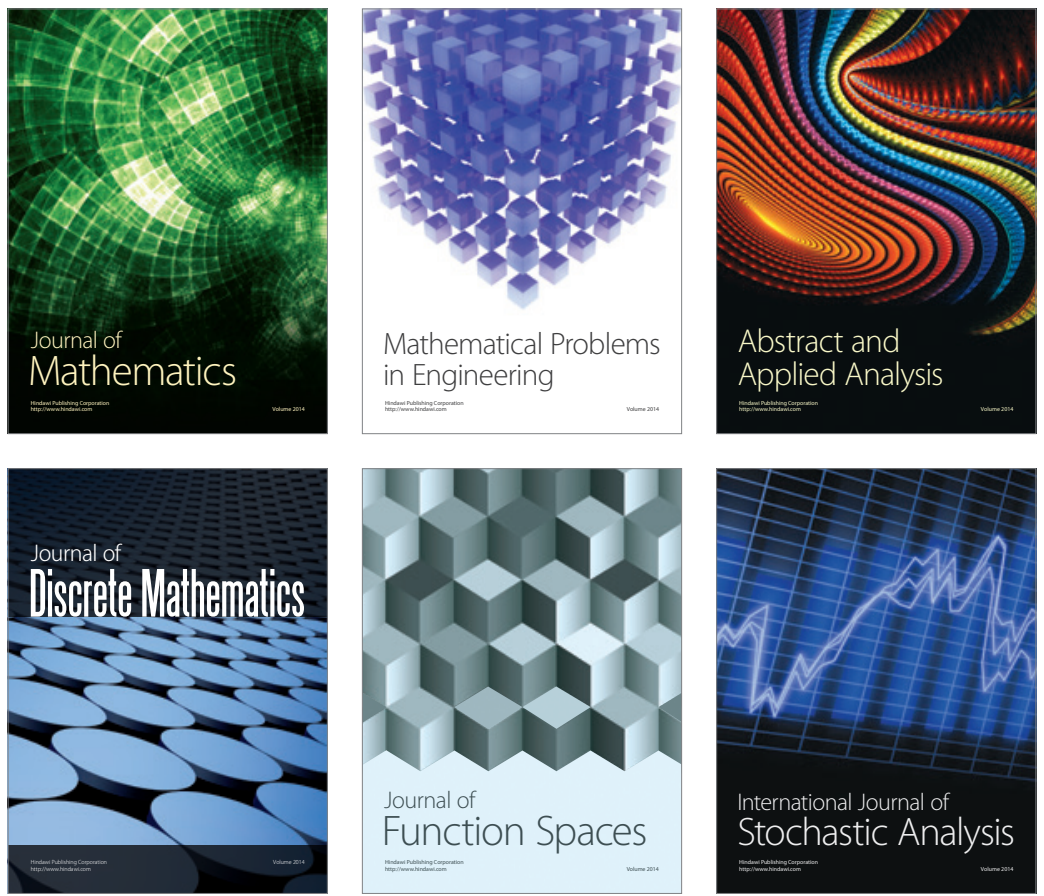

Journal of

Function Spaces

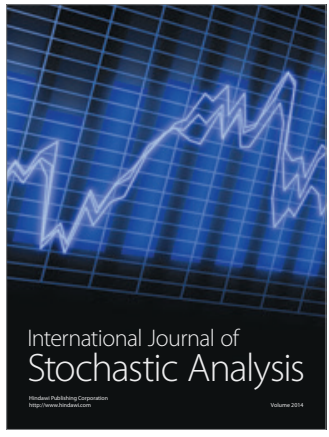

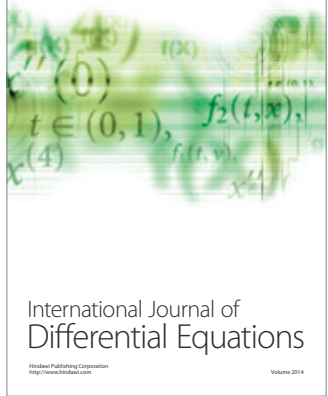
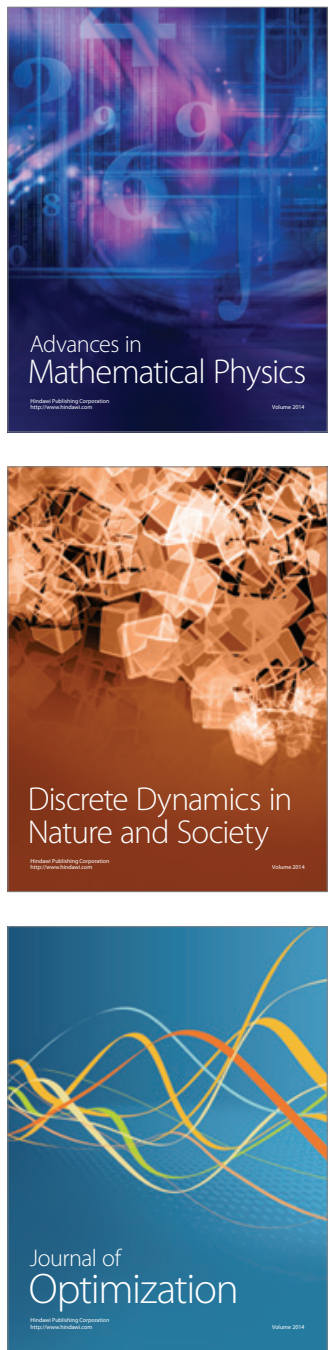